\title{
ALLEVIATION OF Zinnia elegans Jacq. SEED DETERIORATION USING HYDROGEN PEROXIDE AND ORGANIC ACIDS
}

\author{
OGRANICZENIE NEGATYWNYCH EFEKTÓW STARZENIA NASION \\ Zinnia elegans Jacq. POPRZEZ TRAKTOWANIE NADTLENKIEM WODORU \\ I KWASAMI ORGANICZNYMI
}

\begin{abstract}
The decrease of seed quality during storage is strongly associated with damage caused by free radicals. Some compounds of natural origin, known due to their antioxidative and antifungal properties, may be potentially used in organic production for seed treatment as an alternative to pesticides. The aim of the experiment was to study an ability of hydrogen peroxide and organic acid to improve germination, vigour and health of non-deteriorated and deteriorated zinnia seeds. Two seed samples, cv. Illumination and Orys, varying in initial infestation with fungi were tested. For deterioration seeds were kept at $30^{\circ} \mathrm{C}$ and $80 \% \mathrm{RH}$ for 30 days. Seed quality tests were performed before and after deterioration for controls and seeds soaked in $3.0 \%$ hydrogen peroxide solution and in 1.0 and 5.0\% solutions of ascorbic and lactic acids for 10, 30 and 60 min. The controls were untreated seeds, seeds soaked in $0.2 \%$ solution of Kaptan zawiesinowy 50 WP for 30 min and seeds soaked in distilled water for 10, 30 and 60 min. Treating zinnia seeds with organic acids more significantly affected seed germination and health after deterioration than before, and improvement of germination capacity was usually correlated with decrease of the percentage of abnormal diseased seedlings. Deterioration had no influence on mean germination time, whereas in particular cases treating seeds with hydrogen peroxide and organic acids negatively affected this parameter. After deterioration regardless of treatment increased number of seeds free from fungi. Lactic acid followed by hydrogen peroxide and ascorbic acid effectively limited growth of A. alternata, A. zinnia and Fusarium spp. on zinnia seeds, however at higher concentration negatively affected seed germination and vigour. Moreover, treating seeds with hydrogen peroxide and organic acids many a time increased seeds infestation with $B$. cinerea.
\end{abstract}

Keywords: ascorbic acid, lactic acid, hydrogen peroxide, seed deterioration, zinnia

Zinnia (Zinnia elegans Jacq.), cultivated usually for cut flowers and flowerbeds, belongs to the worldwide most popular annual ornamental plants. For sever damages of zinnia plants in the field in most cases responsible are Alternaria zinniae, Botrytis cinerea, Fusarium culmorum, F. equiseti and Sclerotinia sclerotiorum [1, 2]. Furthermore, Alternaria alternata, A. zinniae, Botrytis cinerea, Fusarium spp. and Penicillium spp. were frequently detected in zinnia seeds [3,4]. A. zinniae has been considered the most important fungal seedborne pathogen of zinnia, causing spotting of petals, foliage and stems as well as

\footnotetext{
${ }^{1}$ Department of Phytopathology, Seed Science and Technology, Poznan University of Life Sciences, Baranowo, ul. Szamotulska 28, 62-081 Przeźmierowo, Poland, email: dorota.szopinska@up.poznan.pl
} 
rotting of roots $[2,5]$. In individual years, almost all zinnia seeds in a lot may be infected by this pathogen $[3,4,6]$.

One of the most important aspects of organic crop production is abandoning of pesticides [7]. For this reason, in organic agriculture particularly difficult seems to be controlling diseases at every stage of production. The first step of the control, necessary to avoid transmission of seedborne pathogens, is prevention. Chemical compounds of natural origin, which are traditionally used in food industry as safe for human and environment, may be potentially used in organic production for seed treatment as an alternative to pesticides. Within the plant, some of those chemicals play many crucial roles in growth and metabolism.

In cell metabolism many toxic reactive oxygen species are formed naturally. Seed deterioration is strongly associated with damage caused by free radicals. The particles caused direct damage to mitochondria, enzyme systems, and chromosomes, resulting in weakened metabolism and the inability to repair genetic damage. They also cause lipid peroxidation, leading to the poor membrane integrity, reduced cellular and sub-cellular compartmentation, and leakage of solutes from the cell [8]. Antioxidants may preserving seed vigour by contributing an electron to neutralise free radicals to non-reactive species. According to Arrigoni and De Tullio [9], efficient antioxidant must be present in an adequate amount in the cell, it must react with a variety of free radicals and it must be suitable for regeneration. Ascorbic acid, possessing all of these properties, is one of the most known antioxidants. Many metabolic reactions influencing crucial physiological processes, such as cell division, gene expression and the activation of biological defence mechanisms are ascorbic acid dependent. Molan [10] reported that the bactericidal action of hydrogen peroxide present naturally in honey can be potentiated by ascorbic acid. It was found that ascorbic acid in a concentration of $2.5 \%$ resulted in a strong decrease of seed associated bacteria without affecting seed germination $[7,11]$.

Much more known are antimicrobial properties of lactic acid bacteria, which are used commonly for bio-preservation of food and feed products [12]. The mechanism of these properties has been intensively studied in recent years [13-18]. Nevertheless, exact chemical nature of the antibacterial and antifungal substances produced by the bacteria has not been revealed yet [14, 15, 19]. De Muynck et al. [15] suggested that for the antifungal activity of lactic acid bacteria primarily responsible are organic acids like: lactic, acetic, 3-phenyl-L-lactic and caproic acids. Lin and Yen [20], which investigated nineteen strains of lactic acid bacteria for antioxidative activity, found that the bacteria demonstrated various degrees of ability for hydroxyl radical scavenge. However, since the intracellular cell-free extract was a mixture, is it hard to judge what exactly contributed to this process. Hydrogen peroxide $\left(\mathrm{H}_{2} \mathrm{O}_{2}\right)$ is toxic molecule due to its highly oxidative reactivity and long life. Nevertheless, $\mathrm{H}_{2} \mathrm{O}_{2}$ has been reported to improve germination of seeds of barley (Hordeum vulgare L.) and wheat (Triticum aestivum L.) [21], eastern gamagrass (Tripsacum dactyloides L.) [22] rice (Oryza sativa L.) [23, 24], watermelon (Citrullus lanatus (Thunb.)) [25, 26], camphor tree (Cinnamomum camphora (L.) J.Presl.) [27], muscadine (Vitis rotundifolia Michx.) [28], zinnia [29] and many other plants. Ogawa and Iwabuchi [29] suggested that hydrogen peroxide promote germination of zinnia seeds by oxidation of germination inhibitors present in the pericarp. Oxidative abilities of $\mathrm{H}_{2} \mathrm{O}_{2}$ are used in food industry for decontaminating fruits and vegetables from human pathogens and for reducing fungi responsible for post-harvest decay during storage [21, 30-32]. 
The aim of this experiment was to test various hydrogen peroxide and organic acid treatments in attempt to improve germination, vigour and health of non-deteriorated and deteriorated zinnia seeds.

\section{Materials and methods}

The seeds of Z. elegans cv Illumination (Sample I) and cv Orys (Sample II), obtained from Seed Company CNOS-PNOS in Poznań, were used in the experiment. The seeds were treated with hydrogen peroxide (Laboratorium Farmaceutyczne Avena S.J.), ascorbic acid (Fluka Chemie GmbH.) and lactic acid (Fluka Chemie $\mathrm{GmbH}$ ). The fungicide Kaptan zawiesinowy 50 WP (a.i. 50\% kaptan), produced by Organika-Azot S.A., were used as an alternative control.

The quality of treated and untreated zinnia seeds before and after deterioration was evaluated. For deterioration seeds of both samples were kept at cotton bags in suboptimal conditions, i.e. $30^{\circ} \mathrm{C}$ and $80 \%$ relative humidity ( $\left.\mathrm{RH}\right)$. After 30 days of deterioration seeds were dried back at $20^{\circ} \mathrm{C}$ and $45 \% \mathrm{RH}$ for $24 \mathrm{~h}$. Deteriorated and non-deteriorated seeds were stored in tight plastic bags at $-20^{\circ} \mathrm{C}$ until used.

Germination, vigour and seed health tests were performed before and after deterioration for controls and seeds soaked in $3.0 \%$ hydrogen peroxide solution and in $1.0 \%$ and $5.0 \%$ solutions of ascorbic and lactic acids for 10, 30 and $60 \mathrm{~min}$. The controls were untreated seeds, seeds soaked in $0.2 \%$ solution of fungicide for $30 \mathrm{~min}$ and seeds soaked in distilled water for 10, 30 and $60 \mathrm{~min}$.

Percentage of normal seedlings (germination capacity) and abnormal diseased seedlings were determined after 4 and 10 days according to the ISTA rules [33]. Six replicates of 50 seeds from each treatment were placed in Petri dishes ( 25 seeds per dish) containing six layers of moistened blotters and incubated in the darkness, at $20^{\circ} \mathrm{C}$. To characterise germination rate, six replicates of 50 seeds were incubated under the same conditions as in the previous test. Radicle protrusion was scored daily for 10 days. The mean germination time (MGT) was evaluated using statistical program Seed Calculator 2.1. [34]. Seed health analysis was performed on 200 seeds from each treatment by the deep freezing blotter method. The seeds were plated on six layers of moistened blotters placed in Petri dishes at the rate of 10 seeds per dish (four replicates of 50 seeds). The dishes were kept in darkness at $20^{\circ} \mathrm{C}$ for one day, at $-20^{\circ} \mathrm{C}$ for 20 hours and then for eight days at $20^{\circ} \mathrm{C}$, under 12 hours alternating cycles of NUV light and darkness. The number of seeds infested with fungi and seeds free from fungi were determined using a stereo-microscope and compound microscope [35].

The results obtained were compared by means of two-way analysis of variance. Duncan's multiple range test was applied to estimate the difference between the means at a level $\alpha=0.05$.

\section{Results}

Both samples characterized low germination capacity connected with high percentage of abnormal diseased seedlings and dead seeds (Tables 1-3). Soaking seeds in distilled water many a time increased the number of normal seedlings and simultaneously decreased the number of diseased seedlings (Tables 1 and 2). This effect was particularly evident in sample II after seed deterioration. Generally, it was observed in the whole experiment that 
all treatments more significantly affected seed quality parameters after deterioration. Because hydration itself strongly influenced seed characteristics, the results of the treatments with hydrogen peroxide and organic acids were compared with those results of time-corresponding water treatments. Short-time (10 and $30 \mathrm{~min})$ hydrogen peroxide treatment improved significantly germination of non-deteriorated seeds of sample II. However, this beneficial effect was not observed after deterioration. Ascorbic acid at $1 \%$ concentration positively affected germination of deteriorated and non-deteriorated seeds of sample I after 30 and 60 min treatment. After deterioration 5\% ascorbic acid, applied by the same time, also improved the parameter. In sample II shortest duration of treatment favoured germination capacity, regardless of the acid concentration and seed deterioration. Lactic acid at 1 and 5\% concentration applied for longer time (30 and $60 \mathrm{~min}$ ) negatively affected germination of the seeds of sample II. This phenomenon was also observed in sample I, when non-deteriorated seeds were soaked in 5\% lactic acid solution for 60 min. Although, after deterioration the acid applied at 1 and 5\% concentration for 60 and 30 min respectively, increased significantly germination capacity. Deterioration strongly modified effectiveness of hydrogen peroxide and organic acids in limitation of the percentage of abnormal seedlings in both samples (Table 2). Moreover, the number of the seedlings was usually significantly lower after deterioration, and in no case higher than in control (untreated seeds). On average, lower number of abnormal seedlings was observed in sample I after 30 and 60 min treatment with hydrogen peroxide and 1 and 5\% ascorbic acid, and in both samples after soaking seeds in 5\% lactic acid, regardless of the treatment duration. All applied treatments, except fungicide, in a low extent influenced the percentage of dead seeds (Table 3). Nevertheless, the number of dead seeds increased in sample II after treatment with 1 and 5\% lactic acid for 30 and 60 min and with 5\% ascorbic acid for 30 min.

Deterioration had no influence on mean germination time of untreated seeds (Table 4). An improvement of the parameter was observed only when non-deteriorated seeds of samples I and II were soaked in distilled water for 60 and 30 min respectively, and when deteriorated seeds of sample II were soaked for $10 \mathrm{~min}$. Significant prolongation of germination time was observed after 30 min hydrogen peroxide treatment in sample I and in sample II regardless of treatment time. Moreover, this deleterious effect was connected with seeds deterioration. However, lactic acid especially at higher concentration, prolonged germination time of the seeds from both samples, regardless of deterioration. The same effect was found in sample II, when seeds were treated with 5\% ascorbic acid. Other variants of seed treatment had no effect on the parameter.

On average, number of seeds free from fungi was significantly higher after deterioration (Table 5). Soaking seeds in distilled water alone as well as ascorbic acid treatment had no influence on total seeds infestation. However, treating seeds in hydrogen peroxide and lactic acid solutions resulted in significant increase of a number of seeds free from fungi, especially after deterioration. Moreover, soaking seeds of sample II in 5\% lactic acid affected total infestation with fungi even in a higher extent than fungicide treatment. This phenomenon was observed often separately for each examined fungus (Tables 6-9). Among 30 species and genera of fungi detected on the seeds of both samples Alternaria alternata (Fr.) Keissler, A. zinniae M. B. Ellis, Botrytis cinerea Pers. ex Fr. and Fusarium spp. were examined in detail, due to their common occurrence and/or potential pathogenicity. On average the number of seeds infested with each of those fungi decreased 
significantly after deterioration. Moreover, lactic acid followed by hydrogen peroxide many a time limited growth of Alternaria spp. and Fusarium spp. more effectively than ascorbic acid. Incidence of $A$. alternata in sample I and II were significantly reduced by hydrogen peroxide and 1 and 5\% lactic acid treatment regardless of treatment time (Table 6). The phenomenon was observed in deteriorated seeds of both samples and in non-deteriorated seeds of sample II. In non-deteriorated seeds of sample I presence of A. alternata was limited only after 30 and 60 min treatment with hydrogen peroxide and after soaking seeds in 5\% lactic acid. Moreover, treating seeds of both samples with lactic acid in higher concentration was frequently more efficient than fungicide treatment. Ascorbic acid on the other hand, either increased initially very high infestation or had no effect on the number of seeds infested with the fungus. Opposite results were observed in case of $A$. zinniae, where soaking seeds in ascorbic acid solutions very often limited incidence of the pathogen, especially after seed deterioration (Table 7). Moreover, activity of all tested compounds was more visible in sample I, which was showing much higher level of seed infection. As in the case of A. alternata, soaking seeds of sample I an II in lactic acid solutions, especially at higher concentration, positively reduced infection with $A$. zinniae. The effectiveness of hydrogen peroxide was generally smaller, and in sample II was mainly observed after seed deterioration. Untreated seeds of sample II characterized low $2.5 \%$ infection with $B$. cinerea and seeds of sample II were not infested with this fungus (Table 8). Generally, treating seeds of both samples with hydrogen peroxide and organic acids negatively affected seed infestation with $B$. cinerea. Lactic acid at $1 \%$ concentration applied for 60 min increased the number of seeds infested with this pathogen from 2.5 to $33 \%$ in sample I. However, in sample II, $24.5 \%$ seeds infested with the fungus were observed after 60 min treatment in 5\% ascorbic acid solution. Only treatment with 5\% lactic acid treatment had no effect on an incidence of this pathogen. Generally, deterioration reduced significantly seeds infestation with $B$. cinerea in both samples, regardless of treatment, and many a time the growth of the fungus was completely suppressed. The increase of the percentage of seeds infected with the pathogen was observed only after soaking deteriorated seeds of sample I in 5\% ascorbic acid for $60 \mathrm{~min}$. The untreated seeds of the both tested samples were severely infested with Fusarium spp. (Table 9). Hydrogen peroxide as well as lactic acid treatment effectively limited growth of the fungi on/in non-deteriorated and deteriorated seeds of both samples, whereas ascorbic acid treatment, effectively controlled seeds infestation with Fusarium spp. only after deterioration. As in the previous cases lactic acid at 5\% concentration most effectively control the fungi, and many a time, especially if the seeds were treated longer than $10 \mathrm{~min}$, protected the seeds more efficiently than fungicide. 
Effect of zinnia seed deterioration and treatment with hydrogen peroxide and organic acids on seed germination capacity

\begin{tabular}{|c|c|c|c|c|c|c|}
\hline \multirow{2}{*}{ Treatment } & \multicolumn{6}{|c|}{ Germination capacity [\%] } \\
\hline & \multicolumn{2}{|c|}{ Non-deteriorated } & \multicolumn{2}{|c|}{ Deteriorated } & \multicolumn{2}{|c|}{ Average } \\
\hline \multicolumn{7}{|c|}{ Sample I } \\
\hline Control* & 34.3 & a-d** & 27.7 & $\mathrm{a}-\mathrm{c}$ & 31.0 & A \\
\hline Fungicide & 45.0 & $\mathrm{~d}-\mathrm{k}$ & 46.3 & d-m & 45.7 & E-G \\
\hline $\mathrm{W} 10 \mathrm{~min}$ & 40.0 & $d-i$ & 46.7 & e-m & 43.4 & C-G \\
\hline $\mathrm{W} 30 \mathrm{~min}$ & 36.0 & b-e & 36.3 & b-e & 36.2 & $\mathrm{~A}-\mathrm{C}$ \\
\hline $\mathrm{W} 60 \mathrm{~min}$ & 43.3 & $d-j$ & 35.7 & b-e & 39.5 & B-E \\
\hline $\mathrm{H}_{2} \mathrm{O}_{2} 3 \% 10 \mathrm{~min}$ & 42.3 & $\mathrm{~d}-\mathrm{j}$ & 38.7 & d-h & 40.5 & B-F \\
\hline $\mathrm{H}_{2} \mathrm{O}_{2} 3 \% 30 \mathrm{~min}$ & 49.3 & d-m & 42.0 & $d-j$ & 45.7 & D-G \\
\hline $\mathrm{H}_{2} \mathrm{O}_{2} 3 \% 60 \mathrm{~min}$ & 52.0 & i-n & 43.3 & $d-j$ & 47.7 & F-H \\
\hline $\mathrm{AA} 1 \% 10 \mathrm{~min}$ & 37.0 & $c-f$ & 37.3 & $\mathrm{c}-\mathrm{g}$ & 37.2 & A-D \\
\hline AA $1 \% 30 \mathrm{~min}$ & 57.0 & $1-n$ & 53.3 & $j-n$ & 55.2 & I \\
\hline AA $1 \% 60 \mathrm{~min}$ & 59.0 & $\mathrm{n}$ & 49.7 & h-n & 54.4 & $\mathrm{HI}$ \\
\hline AA $5 \% 10 \mathrm{~min}$ & 35.7 & $b-e$ & 47.0 & e-m & 41.4 & B-G \\
\hline AA $5 \% 30 \mathrm{~min}$ & 41.0 & $d-i$ & 56.7 & $1-n$ & 48.9 & G-I \\
\hline AA $5 \% 60 \mathrm{~min}$ & 42.7 & $d-j$ & 49.3 & g-n & 46.0 & E-G \\
\hline LA $1 \% 10 \mathrm{~min}$ & 47.0 & $e-m$ & 45.0 & $\mathrm{~d}-\mathrm{k}$ & 46.0 & E-G \\
\hline LA $1 \% 30 \mathrm{~min}$ & 43.7 & $d-j$ & 47.0 & e-m & 45.4 & E-G \\
\hline LA $1 \% 60 \mathrm{~min}$ & 40.0 & $d-i$ & 48.7 & $f-n$ & 44.4 & D-G \\
\hline LA $5 \% 10 \mathrm{~min}$ & 38.0 & $c-h$ & 59.3 & $k-n$ & 48.7 & E-G \\
\hline LA $5 \% 30 \mathrm{~min}$ & 27.0 & $a b$ & 57.3 & $\mathrm{mn}$ & 42.2 & B-G \\
\hline LA $5 \% 60 \mathrm{~min}$ & 25.7 & $\mathrm{a}$ & 45.7 & $d-1$ & 35.7 & $\mathrm{AB}$ \\
\hline Average & 41.8 & A & 45.7 & $\mathrm{~B}$ & & \\
\hline \multicolumn{7}{|c|}{ Sample II } \\
\hline Control & 36.0 & $a-c$ & 40.7 & b-d & 38.4 & $\mathrm{~B}$ \\
\hline Fungicide & 61.0 & h-k & 62.0 & $\mathrm{i}-\mathrm{m}$ & 61.5 & $\mathrm{EF}$ \\
\hline $\mathrm{W} 10 \mathrm{~min}$ & 41.3 & b-d & 58.3 & $h-j$ & 49.8 & $\mathrm{CD}$ \\
\hline W $30 \mathrm{~min}$ & 52.3 & $e-i$ & 66.0 & $j-n$ & 59.2 & $\mathrm{EF}$ \\
\hline $\mathrm{W} 60 \mathrm{~min}$ & 57.7 & $h-j$ & 61.3 & h-1 & 59.5 & $\mathrm{EF}$ \\
\hline $\mathrm{H}_{2} \mathrm{O}_{2} 3 \% 10$ min & 65.7 & $j-n$ & 61.3 & h-1 & 63.5 & $\mathrm{~F}$ \\
\hline $\mathrm{H}_{2} \mathrm{O}_{2} 3 \% 30 \mathrm{~min}$ & 69.7 & $\mathrm{k}-\mathrm{n}$ & 62.3 & $\mathrm{i}-\mathrm{m}$ & 66.0 & $\mathrm{~F}$ \\
\hline $\mathrm{H}_{2} \mathrm{O}_{2} 3 \% 60 \mathrm{~min}$ & 62.7 & $\mathrm{i}-\mathrm{m}$ & 65.7 & $j-n$ & 64.2 & $\mathrm{~F}$ \\
\hline $\mathrm{AA} 1 \% 10 \mathrm{~min}$ & 53.3 & $e-i$ & 70.0 & $\mathrm{k}-\mathrm{n}$ & 61.7 & $\mathrm{EF}$ \\
\hline AA $1 \% 30 \mathrm{~min}$ & 59.3 & $h-j$ & 71.7 & $1-n$ & 65.5 & $\mathrm{~F}$ \\
\hline AA $1 \% 60 \mathrm{~min}$ & 45.3 & $b-f$ & 74.3 & $\mathrm{n}$ & 59.8 & $\mathrm{EF}$ \\
\hline AA $5 \% 10 \mathrm{~min}$ & 57.7 & $h-j$ & 72.0 & $\mathrm{mn}$ & 64.9 & $\mathrm{~F}$ \\
\hline AA $5 \% 30 \mathrm{~min}$ & 55.0 & $f-j$ & 62.7 & $\mathrm{i}-\mathrm{m}$ & 58.9 & $\mathrm{EF}$ \\
\hline AA $5 \% 60 \mathrm{~min}$ & 53.7 & $f-i$ & 57.3 & $g-j$ & 55.5 & DE \\
\hline LA $1 \% 10 \mathrm{~min}$ & 49.7 & $c-f$ & 50.3 & $d-h$ & 50.0 & $\mathrm{C}$ \\
\hline LA $1 \% 30 \mathrm{~min}$ & 51.0 & d-h & 46.7 & d-g & 48.9 & $\mathrm{CD}$ \\
\hline LA $1 \% 60 \mathrm{~min}$ & 53.3 & $e-i$ & 42.7 & $b-e$ & 48.0 & $\mathrm{C}$ \\
\hline LA $5 \% 10 \mathrm{~min}$ & 35.3 & $a-c$ & 58.3 & $h-j$ & 46.8 & $\mathrm{C}$ \\
\hline LA $5 \% 30 \mathrm{~min}$ & 35.0 & $a b$ & 43.0 & $b-e$ & 39.0 & $\mathrm{~B}$ \\
\hline LA $5 \% 60 \mathrm{~min}$ & 27.3 & $\mathrm{a}$ & 29.7 & $\mathrm{a}$ & 28.5 & A \\
\hline Average & 51.1 & $\mathrm{~A}$ & 57.8 & $\mathrm{~B}$ & & \\
\hline
\end{tabular}

*Control - untreated seeds; Fungicide - seeds soaked for 30 min in $0.2 \%$ solution of Kaptan zawiesinowy 50 WP; W 10 min, W 30 min, W 60 min - seeds soaked in distilled water for respectively 10, 30 and $60 \mathrm{~min}^{\circ} \mathrm{H}_{2} \mathrm{O}_{2} 3 \%$ 10 min, $\mathrm{H}_{2} \mathrm{O}_{2} 3 \% 30 \mathrm{~min}, \mathrm{H}_{2} \mathrm{O}_{2} 3 \% 60$ min - seeds soaked in $3.0 \%$ hydrogen peroxide for respectively 10,30 and $60 \mathrm{~min}$; AA $1 \% 10 \mathrm{~min}$, AA $1 \% 30 \mathrm{~min}$, AA $1 \% 60 \mathrm{~min}$ - seeds soaked in $1.0 \%$ ascorbic acid for respectively 10 , 30 and $60 \mathrm{~min}$; AA 5\% $10 \mathrm{~min}$, AA 5\% $30 \mathrm{~min}$, AA 5\% $60 \mathrm{~min}$ - seeds soaked in 5.0\% ascorbic acid for respectively 10,30 and $60 \mathrm{~min}$; LA $1 \% 10 \mathrm{~min}$, LA $1 \% 30 \mathrm{~min}$, LA $1 \% 60 \mathrm{~min}$ - seeds soaked in $1.0 \%$ lactic acid 
for respectively 10, 30 and $60 \mathrm{~min}$; LA 5\% $10 \mathrm{~min}$, LA 5\% $30 \mathrm{~min}$, LA $5 \% 60 \mathrm{~min}$ - seeds soaked in 5.0\% lactic acid for respectively 10, 30 and $60 \mathrm{~min}$

** Means followed by the same letter, separately for each sample, are not significantly different from each other, according to Duncan's test $(\alpha=0.05)$

Table 2

Effect of zinnia seed deterioration and treatment with hydrogen peroxide and organic acids on the number of abnormal diseased seedlings

\begin{tabular}{|c|c|c|c|c|c|c|}
\hline \multirow{2}{*}{ Treatment } & \multicolumn{6}{|c|}{ Abnormal diseased seedlings [\%] } \\
\hline & \multicolumn{2}{|c|}{ Non-deteriorated } & \multicolumn{2}{|c|}{ Deteriorated } & \multicolumn{2}{|c|}{ Average } \\
\hline \multicolumn{7}{|c|}{ Sample I } \\
\hline Control & 49.3 & $o-q$ & 51.0 & $\mathrm{pq}$ & 50.2 & $\mathrm{G}$ \\
\hline Fungicide & 36.7 & $h-n$ & 29.7 & d-1 & 33.2 & D-F \\
\hline $\mathrm{W} 10 \mathrm{~min}$ & 45.7 & $n-q$ & 29.3 & d-1 & 37.5 & $\mathrm{EF}$ \\
\hline W $30 \mathrm{~min}$ & 49.7 & $\mathrm{o-q}$ & 29.3 & $\mathrm{c}-\mathrm{k}$ & 39.5 & $\mathrm{~F}$ \\
\hline W $60 \mathrm{~min}$ & 43.3 & $m-q$ & 32.3 & $f-m$ & 37.8 & $\mathrm{EF}$ \\
\hline $\mathrm{H}_{2} \mathrm{O}_{2} 3 \% 10 \mathrm{~min}$ & 37.3 & i-n & 31.7 & e-1 & 34.5 & D-F \\
\hline $\mathrm{H}_{2} \mathrm{O}_{2} 3 \% 30$ min & 25.3 & b-g & 26.0 & b-h & 25.7 & A-C \\
\hline $\mathrm{H}_{2} \mathrm{O}_{2} 3 \% 60 \mathrm{~min}$ & 25.0 & $b-g$ & 24.7 & $b-g$ & 24.9 & $\mathrm{~A}-\mathrm{C}$ \\
\hline $\mathrm{AA} 1 \% 10 \mathrm{~min}$ & 46.3 & $n-q$ & 33.0 & $g-m$ & 39.7 & $\mathrm{~F}$ \\
\hline AA $1 \% 30 \mathrm{~min}$ & 20.7 & a-d & 18.3 & $\mathrm{ab}$ & 19.5 & A \\
\hline AA $1 \% 60 \mathrm{~min}$ & 19.0 & $a-c$ & 27.7 & $b-i$ & 23.4 & $\mathrm{AB}$ \\
\hline AA $5 \% 10 \mathrm{~min}$ & 53.0 & $\mathrm{q}$ & 27.3 & $b-i$ & 40.2 & $\mathrm{~F}$ \\
\hline AA $5 \% 30 \mathrm{~min}$ & 44.3 & $n-q$ & 19.7 & $a-d$ & 32.0 & C-E \\
\hline AA $5 \% 60 \mathrm{~min}$ & 38.0 & i-n & 21.7 & $a-e$ & 29.9 & B-D \\
\hline LA $1 \% 10 \mathrm{~min}$ & 37.3 & i-n & 31.0 & e-1 & 34.2 & D-F \\
\hline LA $1 \% 30 \mathrm{~min}$ & 39.7 & $k-p$ & 28.0 & $b-j$ & 33.9 & D-F \\
\hline LA $1 \% 60 \mathrm{~min}$ & 39.0 & $\mathrm{j}-\mathrm{O}$ & 22.0 & $a-f$ & 30.5 & C-E \\
\hline LA $5 \% 10 \mathrm{~min}$ & 37.7 & i-n & 14.3 & $\mathrm{a}$ & 26.0 & A-C \\
\hline LA $5 \% 30 \mathrm{~min}$ & 32.3 & e-m & 19.7 & $a-d$ & 26.0 & A-C \\
\hline LA $5 \% 60 \mathrm{~min}$ & 40.3 & $1-p$ & 19.0 & $a-c$ & 29.7 & B-D \\
\hline Average & 38.0 & $\mathrm{~B}$ & 26.8 & $\mathrm{~A}$ & & \\
\hline \multicolumn{7}{|c|}{ Sample II } \\
\hline Control & 43.0 & $\mathrm{qr}$ & 43.7 & $\mathrm{r}$ & 43.4 & $\mathrm{G}$ \\
\hline Fungicide & 22.3 & $j-n$ & 14.0 & $d-i$ & 18.2 & B-D \\
\hline $\mathrm{W} 10 \mathrm{~min}$ & 39.7 & $\mathrm{qr}$ & 11.3 & $c-h$ & 25.5 & $\mathrm{EF}$ \\
\hline W $30 \mathrm{~min}$ & 25.3 & $\mathrm{k}-\mathrm{p}$ & 13.0 & $d-i$ & 19.2 & B-E \\
\hline W $60 \mathrm{~min}$ & 24.7 & $\mathrm{k}-\mathrm{o}$ & 16.0 & $\mathrm{f}-\mathrm{k}$ & 20.4 & C-E \\
\hline $\mathrm{H}_{2} \mathrm{O}_{2} 3 \% 10 \mathrm{~min}$ & 20.0 & $\mathrm{i}-\mathrm{m}$ & 18.7 & h-1 & 19.4 & C-E \\
\hline $\mathrm{H}_{2} \mathrm{O}_{2} 3 \% 30 \mathrm{~min}$ & 15.3 & $e-j$ & 16.0 & $e-j$ & 15.7 & $\mathrm{BC}$ \\
\hline $\mathrm{H}_{2} \mathrm{O}_{2} 3 \% 60 \mathrm{~min}$ & 21.3 & $\mathrm{i}-\mathrm{m}$ & 8.3 & $b-f$ & 14.8 & $\mathrm{BC}$ \\
\hline $\mathrm{AA} 1 \% 10 \mathrm{~min}$ & 33.7 & o-r & 8.0 & $b-d$ & 20.9 & B-E \\
\hline AA $1 \% 30 \mathrm{~min}$ & 28.3 & $\mathrm{~m}-\mathrm{p}$ & 8.3 & b-e & 18.3 & B-D \\
\hline AA $1 \% 60 \mathrm{~min}$ & 42.3 & $\mathrm{qr}$ & 4.7 & $\mathrm{ab}$ & 23.5 & C-E \\
\hline $\mathrm{AA} 5 \% 10 \mathrm{~min}$ & 27.3 & $1-p$ & 10.7 & $c-h$ & 19.0 & B-E \\
\hline AA $5 \% 30 \mathrm{~min}$ & 32.3 & $n-q$ & 10.3 & $c-h$ & 21.3 & C-E \\
\hline AA $5 \% 60 \mathrm{~min}$ & 34.3 & o-r & 8.7 & $b-f$ & 21.5 & C-E \\
\hline LA $1 \% 10 \mathrm{~min}$ & 26.3 & $1-p$ & 32.0 & $n-q$ & 29.2 & $\mathrm{~F}$ \\
\hline LA $1 \% 30 \mathrm{~min}$ & 20.3 & $\mathrm{i}-\mathrm{m}$ & 27.0 & $1-p$ & 23.7 & D-F \\
\hline LA $1 \% 60 \mathrm{~min}$ & 20.3 & $\mathrm{i}-\mathrm{m}$ & 35.7 & p-r & 28.0 & $\mathrm{~F}$ \\
\hline LA $5 \% 10 \mathrm{~min}$ & 17.3 & g-k & 10.0 & b-g & 13.7 & B \\
\hline LA $5 \% 30 \mathrm{~min}$ & 11.3 & $\mathrm{c}-\mathrm{h}$ & 6.0 & $\mathrm{bc}$ & 8.7 & A \\
\hline LA $5 \% 60 \mathrm{~min}$ & 9.7 & b-g & 2.0 & $\mathrm{a}$ & 5.9 & A \\
\hline Average & 25.8 & $\mathrm{~B}$ & 15.2 & $\mathrm{~A}$ & & \\
\hline
\end{tabular}

For explanation see Table 1 
Table 3

Effect of zinnia seed deterioration and treatment with hydrogen peroxide and organic acids on the number of dead seeds

\begin{tabular}{|c|c|c|c|c|c|c|}
\hline \multirow{2}{*}{ Treatment } & \multicolumn{6}{|c|}{ Dead seeds [\%] } \\
\hline & \multicolumn{2}{|c|}{ Non-deteriorated } & \multicolumn{2}{|c|}{ Deteriorated } & \multicolumn{2}{|c|}{ Average } \\
\hline \multicolumn{7}{|c|}{ Sample I } \\
\hline Control & 15.7 & b-h & 21.3 & e-m & 18.5 & B-E \\
\hline Fungicide & 11.7 & $\mathrm{a}-\mathrm{c}$ & 8.0 & $\mathrm{a}$ & 9.9 & A \\
\hline $\mathrm{W} 10 \mathrm{~min}$ & 14.0 & b-e & 18.0 & $b-j$ & 16.0 & $\mathrm{~B}$ \\
\hline $\mathrm{W} 30 \mathrm{~min}$ & 12.7 & $a-d$ & 28.0 & $\mathrm{k}-\mathrm{m}$ & 20.4 & B-F \\
\hline $\mathrm{W} 60 \mathrm{~min}$ & 13.3 & b-e & 28.0 & $\operatorname{lm}$ & 20.7 & B-F \\
\hline $\mathrm{H}_{2} \mathrm{O}_{2} 3 \% 10 \mathrm{~min}$ & 19.0 & $\mathrm{c}-1$ & 28.7 & $\operatorname{lm}$ & 23.9 & D-F \\
\hline $\mathrm{H}_{2} \mathrm{O}_{2} 3 \% 30 \mathrm{~min}$ & 21.7 & $f-m$ & 29.0 & $\mathrm{~m}$ & 25.4 & $\mathrm{~F}$ \\
\hline $\mathrm{H}_{2} \mathrm{O}_{2} 3 \% 60 \mathrm{~min}$ & 19.7 & d-m & 28.3 & $\operatorname{lm}$ & 24.0 & $\mathrm{EF}$ \\
\hline $\mathrm{AA} 1 \% 10 \mathrm{~min}$ & 16.7 & $b-i$ & 22.0 & $f-m$ & 19.4 & B-E \\
\hline AA $1 \% 30 \mathrm{~min}$ & 22.0 & $\mathrm{f}-\mathrm{m}$ & 24.3 & $\mathrm{i}-\mathrm{m}$ & 23.2 & C-F \\
\hline AA $1 \% 60 \mathrm{~min}$ & 22.0 & $\mathrm{f}-\mathrm{m}$ & 20.7 & e-m & 21.4 & $\mathrm{C}-\mathrm{F}$ \\
\hline AA $5 \% 10 \mathrm{~min}$ & 11.3 & $a b$ & 20.7 & $e-m$ & 16.0 & B \\
\hline AA $5 \% 30 \mathrm{~min}$ & 14.3 & $b-f$ & 22.0 & $\mathrm{f}-\mathrm{m}$ & 18.2 & B-D \\
\hline AA $5 \% 60 \mathrm{~min}$ & 19.3 & d-1 & 27.0 & $j-m$ & 23.2 & C-F \\
\hline LA $1 \% 10 \mathrm{~min}$ & 15.7 & b-h & 23.0 & g-m & 19.4 & B-E \\
\hline LA $1 \% 30 \mathrm{~min}$ & 16.0 & $b-i$ & 24.3 & h-m & 20.2 & B-F \\
\hline LA $1 \% 60 \mathrm{~min}$ & 18.7 & $c-k$ & 25.0 & i-m & 21.9 & C-F \\
\hline LA $5 \% 10 \mathrm{~min}$ & 16.3 & b-h & 21.0 & e-m & 18.7 & B-E \\
\hline LA $5 \% 30 \mathrm{~min}$ & 26.3 & $j-m$ & 12.3 & $a-d$ & 19.3 & B-E \\
\hline LA $5 \% 60 \mathrm{~min}$ & 14.7 & b-g & 21.7 & e-m & 18.2 & $\mathrm{BC}$ \\
\hline Average & 17.1 & A & 22.7 & $\mathrm{~B}$ & & \\
\hline \multicolumn{7}{|c|}{ Sample II } \\
\hline Control & 10.7 & $d-j$ & 9.0 & d-g & 9.9 & B-D \\
\hline Fungicide & 2.0 & $a b$ & 1.0 & $\mathrm{a}$ & 1.5 & A \\
\hline $\mathrm{W} 10 \mathrm{~min}$ & 8.7 & d-g & 13.7 & $f-j$ & 11.2 & B-E \\
\hline W $30 \mathrm{~min}$ & 15.7 & $\mathrm{~g}-\mathrm{k}$ & 4.3 & $\mathrm{bc}$ & 10.0 & B \\
\hline W $60 \mathrm{~min}$ & 10.0 & $d-i$ & 9.7 & d-h & 9.9 & B-D \\
\hline $\mathrm{H}_{2} \mathrm{O}_{2} 3 \% 10$ min & 5.3 & $\mathrm{~cd}$ & 12.7 & $f-j$ & 9.0 & B \\
\hline $\mathrm{H}_{2} \mathrm{O}_{2} 3 \% 30 \mathrm{~min}$ & 6.7 & c-e & 13.3 & $\mathrm{f}-\mathrm{k}$ & 10.0 & $\mathrm{BC}$ \\
\hline $\mathrm{H}_{2} \mathrm{O}_{2} 3 \% 60 \mathrm{~min}$ & 10.3 & $d-i$ & 11.7 & $f-j$ & 11.0 & B-E \\
\hline AA $1 \% 10 \mathrm{~min}$ & 13.0 & $f-j$ & 7.7 & $d-f$ & 10.4 & B-D \\
\hline AA $1 \% 30 \mathrm{~min}$ & 8.7 & d-g & 11.0 & $e-j$ & 9.9 & $\mathrm{BC}$ \\
\hline AA $1 \% 60 \mathrm{~min}$ & 10.7 & $d-j$ & 8.7 & d-g & 9.7 & $\mathrm{BC}$ \\
\hline AA $5 \% 10 \mathrm{~min}$ & 14.0 & $\mathrm{f}-\mathrm{k}$ & 11.3 & $e-j$ & 12.7 & B-F \\
\hline AA $5 \% 30 \mathrm{~min}$ & 12.7 & $f-j$ & 18.3 & i-1 & 15.5 & $\mathrm{EF}$ \\
\hline AA $5 \% 60 \mathrm{~min}$ & 12.0 & $f-j$ & 17.7 & h-1 & 14.9 & D-F \\
\hline LA $1 \% 10 \mathrm{~min}$ & 13.3 & $\mathrm{f}-\mathrm{k}$ & 17.3 & h-1 & 15.3 & $\mathrm{EF}$ \\
\hline LA $1 \% 30 \mathrm{~min}$ & 17.3 & h-1 & 24.3 & 1 & 20.8 & GH \\
\hline LA $1 \% 60 \mathrm{~min}$ & 15.0 & $f-k$ & 19.0 & $\mathrm{j}-1$ & 17.0 & FG \\
\hline LA $5 \% 10 \mathrm{~min}$ & 18.3 & $\mathrm{j}-1$ & 9.3 & d-g & 13.8 & C-F \\
\hline LA $5 \% 30 \mathrm{~min}$ & 17.7 & $\mathrm{~h}-1$ & 12.0 & $f-j$ & 14.9 & D-F \\
\hline LA $5 \% 60 \mathrm{~min}$ & 26.0 & 1 & 21.7 & $\mathrm{kl}$ & 23.9 & $\mathrm{H}$ \\
\hline Average & 12.4 & $\mathrm{~A}$ & 12.7 & $\mathrm{~A}$ & & \\
\hline
\end{tabular}

For explanation see Table 1 
Table 4

Effect of zinnia seed deterioration and treatment with hydrogen peroxide and organic acids on mean germination time (MGT)

\begin{tabular}{|c|c|c|c|c|c|c|}
\hline \multirow{2}{*}{ Treatment } & \multicolumn{6}{|c|}{ MGT [days] } \\
\hline & \multicolumn{2}{|c|}{ Non-deteriorated } & \multicolumn{2}{|c|}{ Deteriorated } & \multicolumn{2}{|c|}{ Average } \\
\hline \multicolumn{7}{|c|}{ Sample I } \\
\hline Control & 1.68 & b-1 & 1.54 & $a-j$ & 1.61 & A-E \\
\hline Fungicide & 2.05 & $\mathrm{~m}-\mathrm{o}$ & 1.84 & $j-m$ & 1.95 & $\mathrm{HI}$ \\
\hline $\mathrm{W} 10 \mathrm{~min}$ & 1.46 & $a-f$ & 1.63 & $\mathrm{a}-\mathrm{k}$ & 1.55 & A-D \\
\hline W 30 min & 1.40 & $a-c$ & 1.47 & $a-g$ & 1.44 & A \\
\hline W $60 \mathrm{~min}$ & 1.30 & $\mathrm{a}$ & 1.71 & $e-m$ & 1.51 & A-D \\
\hline $\mathrm{H}_{2} \mathrm{O}_{2} 3 \% 10$ min & 1.44 & a-e & 1.97 & $1-n$ & 1.71 & $\mathrm{C}-\mathrm{G}$ \\
\hline $\mathrm{H}_{2} \mathrm{O}_{2} 3 \% 30 \mathrm{~min}$ & 1.52 & $a-i$ & 2.10 & $\mathrm{~m}-\mathrm{o}$ & 1.81 & E-H \\
\hline $\mathrm{H}_{2} \mathrm{O}_{2} 3 \% 60 \mathrm{~min}$ & 1.52 & $\mathrm{a}-\mathrm{g}$ & 1.82 & g-m & 1.67 & A-F \\
\hline $\mathrm{AA} 1 \% 10 \mathrm{~min}$ & 1.74 & d-m & 1.74 & d-1 & 1.74 & D-G \\
\hline AA $1 \% 30 \mathrm{~min}$ & 1.41 & $a-d$ & 1.50 & a-g & 1.46 & $\mathrm{AB}$ \\
\hline AA $1 \% 60 \mathrm{~min}$ & 1.56 & $a-h$ & 1.49 & $a-g$ & 1.53 & A-C \\
\hline AA $5 \% 10 \mathrm{~min}$ & 1.86 & $\mathrm{k}-\mathrm{m}$ & 1.68 & $\mathrm{c}-1$ & 1.77 & E-H \\
\hline AA $5 \% 30 \mathrm{~min}$ & 1.88 & $\mathrm{k}-\mathrm{n}$ & 1.86 & $\mathrm{k}-\mathrm{n}$ & 1.87 & G-I \\
\hline AA $5 \% 60 \mathrm{~min}$ & 2.08 & $\mathrm{~m}-\mathrm{o}$ & 1.66 & b-1 & 1.87 & F-H \\
\hline LA $1 \% 10 \mathrm{~min}$ & 1.72 & d-1 & 1.52 & $a-i$ & 1.62 & A-E \\
\hline LA $1 \% 30 \mathrm{~min}$ & 1.80 & $f-m$ & 1.36 & $a b$ & 1.58 & A-E \\
\hline LA $1 \% 60 \mathrm{~min}$ & 1.84 & h-m & 1.54 & $a-i$ & 1.69 & B-F \\
\hline LA $5 \% 10 \mathrm{~min}$ & 1.85 & i-m & 1.68 & $\mathrm{c}-1$ & 1.77 & E-H \\
\hline LA $5 \% 30 \mathrm{~min}$ & 2.30 & o & 2.05 & $k-n$ & 2.18 & IJ \\
\hline LA $5 \% 60 \mathrm{~min}$ & 2.22 & no & 2.21 & no & 2.22 & $\mathrm{~J}$ \\
\hline Average & 1.74 & $\mathrm{~A}$ & 1.72 & $\mathrm{~A}$ & & \\
\hline \multicolumn{7}{|c|}{ Sample II } \\
\hline Control & 1.09 & $\mathrm{c}$ & 1.08 & $\mathrm{bc}$ & 1.09 & $\mathrm{DE}$ \\
\hline Fungicide & 1.11 & $\mathrm{c}$ & 1.08 & $\mathrm{bc}$ & 1.10 & $\mathrm{E}$ \\
\hline $\mathrm{W} 10 \mathrm{~min}$ & 0.95 & $a-c$ & 0.74 & $\mathrm{a}$ & 0.85 & $\mathrm{AB}$ \\
\hline W $30 \mathrm{~min}$ & 0.81 & $\mathrm{a}$ & 0.88 & $a-c$ & 0.85 & $\mathrm{AB}$ \\
\hline $\mathrm{W} 60 \mathrm{~min}$ & 0.85 & $a-c$ & 0.88 & $a-c$ & 0.87 & $\mathrm{AB}$ \\
\hline $\mathrm{H}_{2} \mathrm{O}_{2} 3 \% 10$ min & 0.95 & $a-c$ & 1.62 & $\mathrm{e}$ & 1.29 & $\mathrm{~F}$ \\
\hline $\mathrm{H}_{2} \mathrm{O}_{2} 3 \% 30 \mathrm{~min}$ & 0.90 & $a-c$ & 1.58 & $\mathrm{e}$ & 1.24 & $\mathrm{~F}$ \\
\hline $\mathrm{H}_{2} \mathrm{O}_{2} 3 \% 60 \mathrm{~min}$ & 1.11 & $\mathrm{bc}$ & 1.61 & $\mathrm{e}$ & 1.36 & FG \\
\hline AA $1 \% 10 \mathrm{~min}$ & 0.89 & $a-c$ & 0.86 & $a-c$ & 0.88 & A-D \\
\hline AA $1 \% 30 \mathrm{~min}$ & 0.98 & $a-c$ & 0.85 & $a-c$ & 0.92 & A-E \\
\hline AA $1 \% 60 \mathrm{~min}$ & 0.97 & $a-c$ & 0.71 & $a b$ & 0.84 & A-D \\
\hline AA $5 \% 10 \mathrm{~min}$ & 0.98 & $a-c$ & 1.04 & $\mathrm{bc}$ & 1.01 & B-E \\
\hline AA $5 \% 30 \mathrm{~min}$ & 0.96 & $a-c$ & 0.94 & $a-c$ & 0.95 & A-E \\
\hline AA $5 \% 60 \mathrm{~min}$ & 1.00 & $a-c$ & 0.87 & $a-c$ & 0.94 & A-C \\
\hline LA $1 \% 10 \mathrm{~min}$ & 0.64 & $\mathrm{a}$ & 0.83 & $a-c$ & 0.74 & A \\
\hline LA $1 \% 30 \mathrm{~min}$ & 0.89 & $a-c$ & 1.01 & $a-c$ & 0.95 & A-E \\
\hline LA $1 \% 60 \mathrm{~min}$ & 1.06 & $\mathrm{bc}$ & 0.95 & $a-c$ & 1.01 & C-E \\
\hline LA $5 \% 10 \mathrm{~min}$ & 1.34 & $\mathrm{~d}$ & 1.62 & $\mathrm{e}$ & 1.48 & $\mathrm{G}$ \\
\hline LA $5 \% 30 \mathrm{~min}$ & 1.88 & $\mathrm{f}$ & 1.95 & $\mathrm{f}$ & 1.92 & I \\
\hline LA $5 \% 60 \mathrm{~min}$ & 1.80 & ef & 1.77 & ef & 1.79 & $\mathrm{H}$ \\
\hline Average & 1.06 & $\mathrm{~A}$ & 1.15 & $\mathrm{~B}$ & & \\
\hline
\end{tabular}

For explanation see Table 1 
Effect of zinnia seed deterioration and treatment with hydrogen peroxide and organic acids on the number of seeds free from fungi

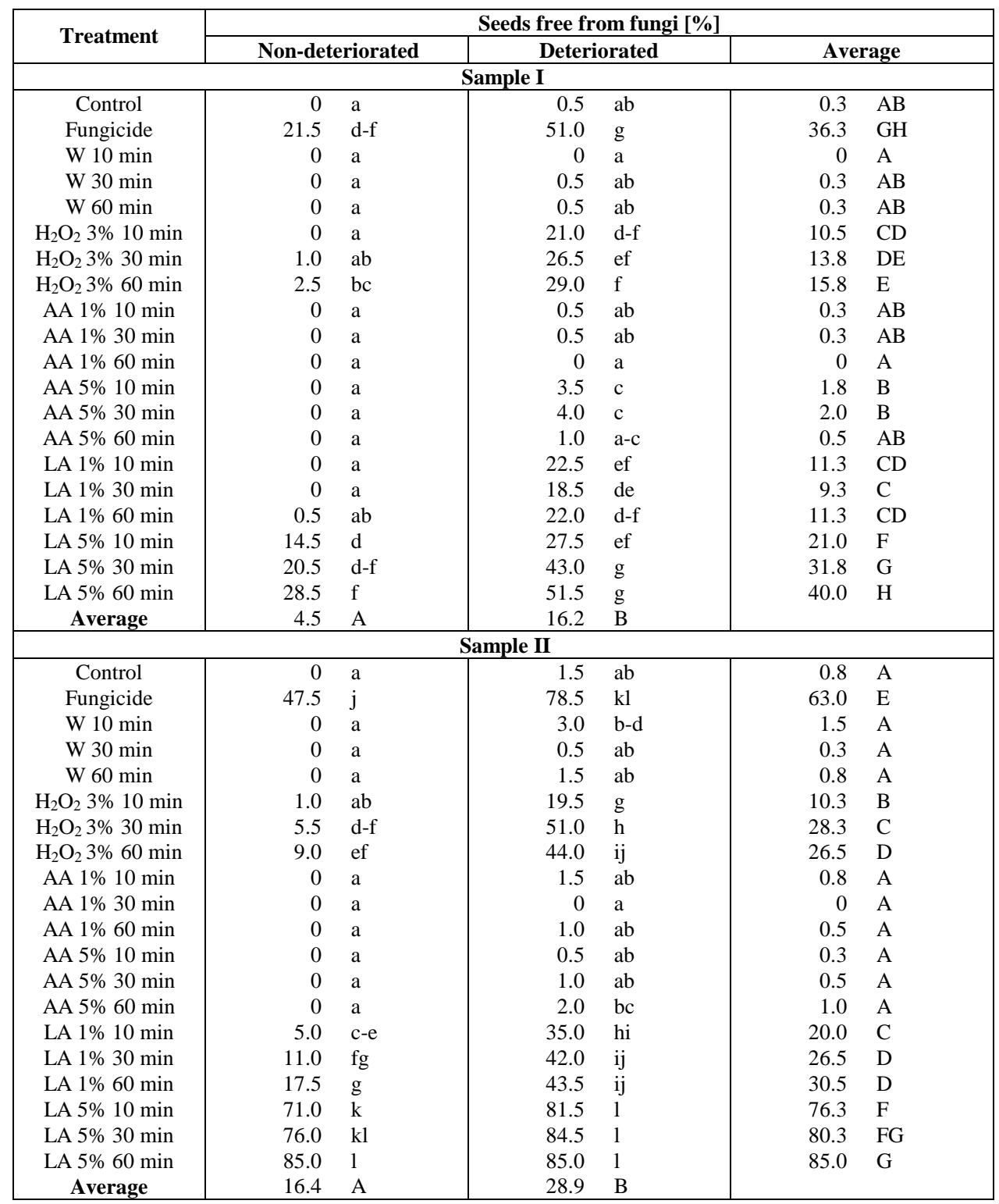

For explanation see Table 1 
Table 6

Effect of zinnia seed deterioration and treatment with hydrogen peroxide and organic acids on seed infestation with Alternaria alternata

\begin{tabular}{|c|c|c|c|c|c|c|}
\hline \multirow{2}{*}{ Treatment } & \multicolumn{6}{|c|}{ Infested seeds [\%] } \\
\hline & \multicolumn{2}{|c|}{ Non-deteriorated } & \multicolumn{2}{|c|}{ Deteriorated } & \multicolumn{2}{|c|}{ Average } \\
\hline \multicolumn{7}{|c|}{ Sample I } \\
\hline Control & 77.5 & $n-q$ & 78.0 & $\mathrm{o}-\mathrm{q}$ & 77.8 & $E$ \\
\hline Fungicide & 59.0 & h-k & 22.0 & $\mathrm{bc}$ & 40.5 & $\mathrm{C}$ \\
\hline $\mathrm{W} 10 \mathrm{~min}$ & 74.0 & m-p & 73.0 & $1-p$ & 73.5 & $\mathrm{E}$ \\
\hline W $30 \mathrm{~min}$ & 79.5 & $o-q$ & 72.0 & $1-p$ & 75.8 & $\mathrm{E}$ \\
\hline W $60 \mathrm{~min}$ & 82.5 & $\mathrm{p}-\mathrm{r}$ & 73.5 & m-p & 78.0 & $\mathrm{E}$ \\
\hline $\mathrm{H}_{2} \mathrm{O}_{2} 3 \% 10 \mathrm{~min}$ & 73.0 & $1-p$ & 36.5 & $d-f$ & 54.8 & $\mathrm{D}$ \\
\hline $\mathrm{H}_{2} \mathrm{O}_{2} 3 \% 30 \mathrm{~min}$ & 53.5 & $g-j$ & 33.0 & c-e & 43.3 & $\mathrm{C}$ \\
\hline $\mathrm{H}_{2} \mathrm{O}_{2} 3 \% 60$ min & 47.0 & f-h & 30.0 & c-e & 38.5 & $\mathrm{C}$ \\
\hline AA $1 \% 10 \mathrm{~min}$ & 79.5 & $o-q$ & 68.0 & $\mathrm{k}-\mathrm{o}$ & 73.8 & $\mathrm{E}$ \\
\hline AA $1 \% 30 \mathrm{~min}$ & 80.5 & $o-q$ & 60.5 & i-1 & 70.5 & $\mathrm{E}$ \\
\hline AA $1 \% 60 \mathrm{~min}$ & 80.5 & $\mathrm{o}-\mathrm{q}$ & 64.0 & $j-m$ & 72.3 & $\mathrm{E}$ \\
\hline AA $5 \% 10 \mathrm{~min}$ & 87.0 & $q-s$ & 64.0 & $j-m$ & 75.5 & $\mathrm{E}$ \\
\hline AA $5 \% 30 \mathrm{~min}$ & 89.5 & $\mathrm{~s}$ & 62.5 & $\mathrm{i}-\mathrm{m}$ & 76.0 & $\mathrm{E}$ \\
\hline AA $5 \% 60 \mathrm{~min}$ & 90.0 & rs & 65.5 & $j-n$ & 77.8 & $\mathrm{E}$ \\
\hline LA $1 \% 10 \mathrm{~min}$ & 83.5 & p-s & 39.5 & ef & 61.5 & $\mathrm{D}$ \\
\hline LA $1 \% 30 \mathrm{~min}$ & 80.5 & $\mathrm{o}-\mathrm{q}$ & 41.5 & e-g & 61.0 & $\mathrm{D}$ \\
\hline LA $1 \% 60 \mathrm{~min}$ & 77.0 & $n-q$ & 37.0 & $d-f$ & 57.0 & $\mathrm{D}$ \\
\hline LA $5 \% 10 \mathrm{~min}$ & 49.5 & $f-i$ & 26.0 & b-d & 37.8 & $\mathrm{BC}$ \\
\hline LA $5 \% 30 \mathrm{~min}$ & 42.5 & e-g & 19.5 & $a b$ & 31.0 & $\mathrm{~B}$ \\
\hline LA $5 \% 60 \mathrm{~min}$ & 33.5 & $c-e$ & 11.5 & $\mathrm{a}$ & 22.5 & A \\
\hline Average & 71.0 & $\mathrm{~B}$ & 48.9 & $\mathrm{~A}$ & & \\
\hline \multicolumn{7}{|c|}{ Sample II } \\
\hline Control & 91.0 & op & 74.5 & $\operatorname{lm}$ & 82.8 & $\mathrm{H}$ \\
\hline Fungicide & 26.5 & ef & 12.0 & $a-c$ & 19.3 & $\mathrm{~B}$ \\
\hline $\mathrm{W} 10 \mathrm{~min}$ & 80.0 & $\mathrm{~m}-\mathrm{o}$ & 63.0 & $\mathrm{jk}$ & 71.5 & $\mathrm{~F}$ \\
\hline W $30 \mathrm{~min}$ & 81.5 & $\mathrm{~m}-\mathrm{o}$ & 64.5 & $\mathrm{j}-1$ & 73.0 & $\mathrm{~F}$ \\
\hline W $60 \mathrm{~min}$ & 85.5 & $n-p$ & 60.5 & $\mathrm{j}$ & 73.0 & $\mathrm{~F}$ \\
\hline $\mathrm{H}_{2} \mathrm{O}_{2} 3 \% 10 \mathrm{~min}$ & 48.0 & hi & 23.0 & fg & 35.5 & $\mathrm{D}$ \\
\hline $\mathrm{H}_{2} \mathrm{O}_{2} 3 \% 30 \mathrm{~min}$ & 47.0 & hi & 30.5 & fg & 38.8 & $\mathrm{D}$ \\
\hline $\mathrm{H}_{2} \mathrm{O}_{2} 3 \% 60$ min & 34.0 & fg & 24.0 & $d-f$ & 29.0 & $\mathrm{C}$ \\
\hline AA $1 \% 10 \mathrm{~min}$ & 93.0 & $\mathrm{p}$ & 76.5 & $\mathrm{~m}$ & 84.8 & $\mathrm{H}$ \\
\hline AA $1 \% 30 \mathrm{~min}$ & 88.5 & $n-p$ & 73.5 & $\mathrm{k}-\mathrm{m}$ & 81.0 & $\mathrm{GH}$ \\
\hline AA $1 \% 60 \mathrm{~min}$ & 91.5 & $\mathrm{p}$ & 81.0 & $\mathrm{mn}$ & 86.3 & $\mathrm{H}$ \\
\hline AA $5 \% 10 \mathrm{~min}$ & 87.5 & $n-p$ & 61.5 & $\mathrm{j}$ & 74.5 & FG \\
\hline AA $5 \% 30 \mathrm{~min}$ & 82.0 & $\mathrm{~m}-\mathrm{o}$ & 59.0 & $\mathrm{j}$ & 70.5 & $\mathrm{~F}$ \\
\hline AA $5 \% 60 \mathrm{~min}$ & 89.5 & $n-p$ & 54.0 & $\mathrm{ij}$ & 71.8 & $\mathrm{~F}$ \\
\hline LA $1 \% 10 \mathrm{~min}$ & 64.0 & $\mathrm{jk}$ & 32.0 & fg & 48.0 & $\mathrm{E}$ \\
\hline LA $1 \% 30 \mathrm{~min}$ & 60.5 & $\mathrm{j}$ & 17.5 & c-e & 39.0 & $\mathrm{D}$ \\
\hline LA $1 \% 60 \mathrm{~min}$ & 41.0 & $\mathrm{gh}$ & 16.0 & $b-d$ & 28.5 & $\mathrm{C}$ \\
\hline LA $5 \% 10 \mathrm{~min}$ & 12.5 & $\mathrm{a}-\mathrm{c}$ & 8.0 & $\mathrm{a}$ & 10.3 & A \\
\hline LA $5 \% 30 \mathrm{~min}$ & 10.0 & $a-c$ & 6.0 & $\mathrm{a}$ & 8.0 & A \\
\hline LA $5 \% 60 \mathrm{~min}$ & 5.5 & $\mathrm{a}$ & 9.0 & $a b$ & 7.3 & A \\
\hline Average & 61.0 & $\mathrm{~B}$ & 42.3 & $\mathrm{~A}$ & & \\
\hline
\end{tabular}

For explanation see Table 1 
Effect of zinnia seed deterioration and treatment with hydrogen peroxide and organic acids on seed infestation with Alternaria zinniae

\begin{tabular}{|c|c|c|c|c|c|c|}
\hline \multirow{2}{*}{ Treatment } & \multicolumn{6}{|c|}{ Infested seeds [\%] } \\
\hline & \multicolumn{2}{|c|}{ Non-deteriorated } & \multicolumn{2}{|c|}{ Deteriorated } & \multicolumn{2}{|c|}{ Average } \\
\hline \multicolumn{7}{|c|}{ Sample I } \\
\hline Control & 64.5 & k-o & 74.0 & op & 69.3 & IJ \\
\hline Fungicide & 40.0 & $c-f$ & 31.5 & $a-d$ & 35.8 & $\mathrm{BC}$ \\
\hline $\mathrm{W} 10 \mathrm{~min}$ & 68.5 & $1-p$ & 79.5 & $\mathrm{p}$ & 74.0 & $\mathrm{~J}$ \\
\hline W $30 \mathrm{~min}$ & 70.0 & $\mathrm{~m}-\mathrm{p}$ & 69.5 & $\mathrm{~m}-\mathrm{p}$ & 69.8 & IJ \\
\hline W $60 \mathrm{~min}$ & 70.5 & $n-p$ & 67.5 & $1-0$ & 69.0 & H-J \\
\hline $\mathrm{H}_{2} \mathrm{O}_{2} 3 \% 10 \mathrm{~min}$ & 76.0 & op & 44.5 & $e-i$ & 60.3 & F-H \\
\hline $\mathrm{H}_{2} \mathrm{O}_{2} 3 \% 30 \mathrm{~min}$ & 56.0 & h-1 & 45.5 & $e-i$ & 50.8 & $\mathrm{DE}$ \\
\hline $\mathrm{H}_{2} \mathrm{O}_{2} 3 \% 60 \mathrm{~min}$ & 54.0 & $e-i$ & 41.5 & $\mathrm{c}-\mathrm{g}$ & 47.8 & $\mathrm{CD}$ \\
\hline $\mathrm{AA} 1 \% 10 \mathrm{~min}$ & 65.5 & $\mathrm{k}-\mathrm{o}$ & 57.0 & $\mathrm{i}-\mathrm{m}$ & 61.3 & F-I \\
\hline AA $1 \% 30 \mathrm{~min}$ & 67.0 & $1-0$ & 60.0 & $j-m$ & 63.5 & G-I \\
\hline AA $1 \% 60 \mathrm{~min}$ & 67.0 & $1-0$ & 53.5 & $\mathrm{~g}-\mathrm{k}$ & 60.3 & FG \\
\hline AA $5 \% 10 \mathrm{~min}$ & 70.0 & $n-p$ & 55.5 & h-1 & 62.8 & G-I \\
\hline AA $5 \% 30 \mathrm{~min}$ & 59.5 & $j-n$ & 50.5 & $f-j$ & 55.0 & E-G \\
\hline AA $5 \% 60 \mathrm{~min}$ & 49.0 & $f-j$ & 57.0 & h-m & 53.0 & $\mathrm{EF}$ \\
\hline LA $1 \% 10 \mathrm{~min}$ & 48.5 & $f-j$ & 38.0 & $b-f$ & 43.3 & $\mathrm{CD}$ \\
\hline LA $1 \% 30 \mathrm{~min}$ & 40.0 & $c-f$ & 43.5 & d-h & 41.8 & $\mathrm{C}$ \\
\hline LA $1 \% 60 \mathrm{~min}$ & 29.0 & $a-c$ & 33.0 & b-e & 31.0 & B \\
\hline LA $5 \% 10 \mathrm{~min}$ & 38.5 & $b-f$ & 33.5 & b-e & 36.0 & $\mathrm{BC}$ \\
\hline LA $5 \% 30 \mathrm{~min}$ & 29.5 & $a-c$ & 26.5 & $a b$ & 28.0 & B \\
\hline LA $5 \% 60 \mathrm{~min}$ & 21.5 & $\mathrm{a}$ & 21.0 & $\mathrm{a}$ & 21.3 & A \\
\hline Average & 54.2 & $\mathrm{~B}$ & 49.1 & $\mathrm{~A}$ & & \\
\hline \multicolumn{7}{|c|}{ Sample II } \\
\hline Control & 7.0 & h-m & 9.0 & $\mathrm{j}-\mathrm{m}$ & 8.0 & F-H \\
\hline Fungicide & 1.0 & $a-d$ & 4.0 & $\mathrm{~d}-\mathrm{i}$ & 2.5 & $\mathrm{BC}$ \\
\hline $\mathrm{W} 10 \mathrm{~min}$ & 10.5 & $1-m$ & 9.5 & $\mathrm{k}-\mathrm{m}$ & 10.0 & $\mathrm{GH}$ \\
\hline W $30 \mathrm{~min}$ & 8.0 & h-m & 5.5 & $e-m$ & 6.8 & E-G \\
\hline W $60 \mathrm{~min}$ & 11.0 & $\mathrm{~m}$ & 11.0 & $\mathrm{~m}$ & 11.0 & $\mathrm{H}$ \\
\hline $\mathrm{H}_{2} \mathrm{O}_{2} 3 \% 10 \mathrm{~min}$ & 9.0 & $\mathrm{j}-\mathrm{m}$ & 3.5 & $d-j$ & 6.3 & E-G \\
\hline $\mathrm{H}_{2} \mathrm{O}_{2} 3 \% 30 \mathrm{~min}$ & 6.5 & g-m & 7.0 & g-m & 6.8 & E-G \\
\hline $\mathrm{H}_{2} \mathrm{O}_{2} 3 \% 60 \mathrm{~min}$ & 6.0 & $\mathrm{f}-\mathrm{m}$ & 4.0 & e-k & 5.0 & D-F \\
\hline $\mathrm{AA} 1 \% 10 \mathrm{~min}$ & 11.0 & $\mathrm{~m}$ & 3.5 & d-h & 7.3 & E-G \\
\hline AA $1 \% 30 \mathrm{~min}$ & 8.5 & $\mathrm{i}-\mathrm{m}$ & 2.5 & $b-e$ & 5.5 & DE \\
\hline AA $1 \% 60 \mathrm{~min}$ & 7.5 & g-m & 3.0 & $d-i$ & 5.3 & D-F \\
\hline AA $5 \% 10 \mathrm{~min}$ & 4.5 & e-k & 5.5 & e-m & 5.0 & D-F \\
\hline AA $5 \% 30 \mathrm{~min}$ & 5.0 & e-m & 3.5 & $d-j$ & 4.3 & $\mathrm{DE}$ \\
\hline AA $5 \% 60 \mathrm{~min}$ & 4.5 & e-1 & 9.0 & $\mathrm{i}-\mathrm{m}$ & 6.8 & E-G \\
\hline LA $1 \% 10 \mathrm{~min}$ & 5.0 & e-1 & 5.0 & $d-j$ & 5.0 & $\mathrm{DE}$ \\
\hline LA $1 \% 30 \mathrm{~min}$ & 4.5 & $d-j$ & 2.0 & $b-f$ & 3.3 & $\mathrm{CD}$ \\
\hline LA $1 \% 60 \mathrm{~min}$ & 5.5 & $e-m$ & 2.5 & $c-g$ & 4.0 & $\mathrm{DE}$ \\
\hline LA $5 \% 10 \mathrm{~min}$ & 1.0 & $a-c$ & 1.0 & $a-d$ & 1.0 & $\mathrm{AB}$ \\
\hline LA $5 \% 30 \mathrm{~min}$ & 0.5 & $a b$ & 1.0 & $a-d$ & 0.8 & $\mathrm{AB}$ \\
\hline LA $5 \% 60 \mathrm{~min}$ & 0 & $\mathrm{a}$ & 0 & $\mathrm{a}$ & 0 & A \\
\hline Average & 5.8 & $\mathrm{~B}$ & 4.6 & $\mathrm{~A}$ & & \\
\hline
\end{tabular}

For explanation see Table 1 
Table 8

Effect of zinnia seed deterioration and treatment with hydrogen peroxide and organic acids on seed infestation with Botrytis cinerea

\begin{tabular}{|c|c|c|c|c|c|c|}
\hline \multirow{2}{*}{ Treatment } & \multicolumn{6}{|c|}{ Infested seeds [\%] } \\
\hline & \multicolumn{2}{|c|}{ Non-deteriorated } & \multicolumn{2}{|c|}{ Deteriorated } & \multicolumn{2}{|c|}{ Average } \\
\hline \multicolumn{7}{|c|}{ Sample I } \\
\hline Control & 2.5 & c-f & 0 & $\mathrm{a}$ & 1.3 & B-D \\
\hline Fungicide & 0 & $\mathrm{a}$ & 0 & $\mathrm{a}$ & 0 & A \\
\hline $\mathrm{W} 10 \mathrm{~min}$ & 3.0 & b-e & 0 & $\mathrm{a}$ & 1.5 & A-D \\
\hline W $30 \mathrm{~min}$ & 1.0 & $a-c$ & 0 & $\mathrm{a}$ & 0.5 & $\mathrm{AB}$ \\
\hline W $60 \mathrm{~min}$ & 1.5 & $a-d$ & 0 & $\mathrm{a}$ & 0.8 & A-C \\
\hline $\mathrm{H}_{2} \mathrm{O}_{2} 3 \% 10 \mathrm{~min}$ & 4.0 & d-g & 0 & $\mathrm{a}$ & 2.0 & B-D \\
\hline $\mathrm{H}_{2} \mathrm{O}_{2} 3 \% 30$ min & 30.0 & $\mathrm{mn}$ & 0 & $\mathrm{a}$ & 15.0 & $\mathrm{GH}$ \\
\hline $\mathrm{H}_{2} \mathrm{O}_{2} 3 \% 60$ min & 13.5 & ij & 0 & $\mathrm{a}$ & 6.8 & $\mathrm{EF}$ \\
\hline $\mathrm{AA} 1 \% 10 \mathrm{~min}$ & 11.0 & $h-j$ & 0.5 & $a b$ & 5.8 & $\mathrm{EF}$ \\
\hline $\mathrm{AA} 1 \% 30 \mathrm{~min}$ & 17.0 & $\mathrm{j}-1$ & 0.5 & $a b$ & 8.8 & FG \\
\hline $\mathrm{AA} 1 \% 60 \mathrm{~min}$ & 8.0 & $g-i$ & 0 & $\mathrm{a}$ & 4.0 & $\mathrm{DE}$ \\
\hline AA $5 \% 10 \mathrm{~min}$ & 16.0 & $\mathrm{jk}$ & 1.5 & $a-c$ & 8.8 & F-H \\
\hline AA $5 \% 30 \mathrm{~min}$ & 23.5 & $\mathrm{k}-\mathrm{m}$ & 1.0 & $a-c$ & 12.3 & $\mathrm{GH}$ \\
\hline AA $5 \% 60 \mathrm{~min}$ & 28.0 & $\mathrm{mn}$ & 8.0 & $g-i$ & 18.0 & I \\
\hline LA $1 \% 10 \mathrm{~min}$ & 24.0 & $1-n$ & 0 & $\mathrm{a}$ & 12.0 & F-H \\
\hline LA $1 \% 30 \mathrm{~min}$ & 22.0 & $\mathrm{k}-\mathrm{m}$ & 0 & $\mathrm{a}$ & 11.0 & F-H \\
\hline LA $1 \% 60 \mathrm{~min}$ & 33.0 & $\mathrm{n}$ & 0 & $\mathrm{a}$ & 16.5 & $\mathrm{H}$ \\
\hline LA $5 \% 10 \mathrm{~min}$ & 5.5 & $e-h$ & 0 & $\mathrm{a}$ & 2.8 & C-E \\
\hline LA $5 \% 30 \mathrm{~min}$ & 7.5 & e-h & 0 & $\mathrm{a}$ & 3.8 & C-E \\
\hline LA $5 \% 60 \mathrm{~min}$ & 7.0 & f-h & 0 & $\mathrm{a}$ & 3.5 & C-E \\
\hline Average & 12.9 & $\mathrm{~B}$ & 0.6 & $\mathrm{~A}$ & & \\
\hline \multicolumn{7}{|c|}{ Sample II } \\
\hline Control & 0 & $\mathrm{a}$ & 0 & $\mathrm{a}$ & 0 & $\mathrm{~A}$ \\
\hline Fungicide & 0 & $\mathrm{a}$ & 0 & $\mathrm{a}$ & 0 & A \\
\hline $\mathrm{W} 10 \mathrm{~min}$ & 0 & $\mathrm{a}$ & 0 & $\mathrm{a}$ & 0 & $\mathrm{~A}$ \\
\hline $\mathrm{W} 30 \mathrm{~min}$ & 0 & $\mathrm{a}$ & 0 & $\mathrm{a}$ & 0 & $\mathrm{~A}$ \\
\hline $\mathrm{W} 60 \mathrm{~min}$ & 0 & $\mathrm{a}$ & 0 & $\mathrm{a}$ & 0 & $\mathrm{~A}$ \\
\hline $\mathrm{H}_{2} \mathrm{O}_{2} 3 \% 10 \mathrm{~min}$ & 2.5 & $b-d$ & 0 & $\mathrm{a}$ & 1.3 & A-C \\
\hline $\mathrm{H}_{2} \mathrm{O}_{2} 3 \% 30 \mathrm{~min}$ & 7.0 & $\mathrm{f}$ & 0.5 & $a b$ & 3.8 & E-F \\
\hline $\mathrm{H}_{2} \mathrm{O}_{2} 3 \% 60 \mathrm{~min}$ & 6.5 & ef & 0 & $\mathrm{a}$ & 3.3 & C-E \\
\hline $\mathrm{AA} 1 \% 10 \mathrm{~min}$ & 1.5 & $a-c$ & 0 & $\mathrm{a}$ & 0.8 & $\mathrm{AB}$ \\
\hline $\mathrm{AA} 1 \% 30 \mathrm{~min}$ & 3.0 & $c-e$ & 0.5 & $a b$ & 1.8 & B-E \\
\hline AA $1 \% 60 \mathrm{~min}$ & 2.5 & $\mathrm{~cd}$ & 0 & $\mathrm{a}$ & 1.3 & B-D \\
\hline $\mathrm{AA} 5 \% 10 \mathrm{~min}$ & 18.0 & hi & 0 & $\mathrm{a}$ & 9.0 & $\mathrm{GH}$ \\
\hline AA $5 \% 30 \mathrm{~min}$ & 12.0 & gh & 0.5 & $a b$ & 6.3 & FG \\
\hline AA $5 \% 60 \mathrm{~min}$ & 24.5 & $\mathrm{i}$ & 0.5 & $a b$ & 12.5 & $\mathrm{H}$ \\
\hline LA $1 \% 10 \mathrm{~min}$ & 4.0 & $d-f$ & 0 & $\mathrm{a}$ & 2.0 & B-E \\
\hline LA $1 \% 30 \mathrm{~min}$ & 6.0 & $\mathrm{f}$ & 0 & $\mathrm{a}$ & 3.0 & C-E \\
\hline LA $1 \% 60 \mathrm{~min}$ & 7.5 & $\mathrm{fg}$ & 0 & $\mathrm{a}$ & 3.8 & D-F \\
\hline LA $5 \% 10 \mathrm{~min}$ & 1.5 & $a-c$ & 0 & $\mathrm{a}$ & 0.8 & $\mathrm{AB}$ \\
\hline LA $5 \% 30 \mathrm{~min}$ & 0 & $\mathrm{a}$ & 0 & $\mathrm{a}$ & 0 & $\mathrm{~A}$ \\
\hline LA $5 \% 60 \mathrm{~min}$ & 0 & $\mathrm{a}$ & 0 & $\mathrm{a}$ & 0 & A \\
\hline Average & 4.8 & $\mathrm{~B}$ & 0.1 & $\mathrm{~A}$ & & \\
\hline
\end{tabular}

For explanation see Table 1 
Effect of zinnia seed deterioration and treatment with hydrogen peroxide and organic acids on seed infestation with Fusarium spp.

\begin{tabular}{|c|c|c|c|c|c|c|}
\hline \multirow{2}{*}{ Treatment } & \multicolumn{6}{|c|}{ Infested seeds [\%] } \\
\hline & \multicolumn{2}{|c|}{ Non-deteriorated } & \multicolumn{2}{|c|}{ Deteriorated } & \multicolumn{2}{|c|}{ Average } \\
\hline \multicolumn{7}{|c|}{ Sample I } \\
\hline Control & 80.5 & o & 46.0 & $\operatorname{lm}$ & 63.3 & G \\
\hline Fungicide & 24.0 & g-i & 5.5 & $b-d$ & 14.8 & $\mathrm{C}$ \\
\hline W 10 min & 94.0 & $\mathrm{p}$ & 54.5 & $\mathrm{mn}$ & 74.3 & I \\
\hline W $30 \mathrm{~min}$ & 80.5 & o & 47.5 & $1 \mathrm{~m}$ & 64.0 & G \\
\hline W $60 \mathrm{~min}$ & 93.5 & $\mathrm{p}$ & 46.0 & $\operatorname{lm}$ & 69.8 & $\mathrm{HI}$ \\
\hline $\mathrm{H}_{2} \mathrm{O}_{2} 3 \% 10 \mathrm{~min}$ & 54.0 & $\mathrm{mn}$ & 20.0 & f-h & 37.0 & E \\
\hline $\mathrm{H}_{2} \mathrm{O}_{2} 3 \% 30 \mathrm{~min}$ & 47.0 & $\operatorname{lm}$ & 12.0 & $d-f$ & 29.5 & $\mathrm{D}$ \\
\hline $\mathrm{H}_{2} \mathrm{O}_{2} 3 \% 60$ min & 61.5 & $\mathrm{n}$ & 6.5 & $b-d$ & 34.0 & $\mathrm{DE}$ \\
\hline AA $1 \% 10 \mathrm{~min}$ & 83.5 & o & 23.5 & $g-i$ & 53.5 & $\mathrm{~F}$ \\
\hline AA $1 \% 30 \mathrm{~min}$ & 84.0 & o & 41.5 & $\mathrm{kl}$ & 62.8 & $\mathrm{G}$ \\
\hline AA $1 \% 60 \mathrm{~min}$ & 93.0 & $\mathrm{p}$ & 33.5 & $\mathrm{i}-\mathrm{k}$ & 63.3 & $\mathrm{GH}$ \\
\hline AA $5 \% 10 \mathrm{~min}$ & 80.5 & o & 23.5 & $\mathrm{~g}-\mathrm{i}$ & 52.0 & $\mathrm{~F}$ \\
\hline AA $5 \% 30 \mathrm{~min}$ & 81.0 & o & 30.0 & $h-j$ & 55.5 & $\mathrm{~F}$ \\
\hline AA $5 \% 60 \mathrm{~min}$ & 81.0 & o & 19.0 & $\mathrm{fg}$ & 50.0 & $\mathrm{~F}$ \\
\hline LA $1 \% 10 \mathrm{~min}$ & 37.5 & $j-1$ & 15.5 & e-g & 26.5 & D \\
\hline LA $1 \% 30 \mathrm{~min}$ & 31.5 & $\mathrm{i}-\mathrm{k}$ & 18.0 & $\mathrm{e}-\mathrm{g}$ & 24.8 & D \\
\hline LA $1 \% 60 \mathrm{~min}$ & 19.5 & fg & 8.0 & $b-d$ & 13.8 & $\mathrm{C}$ \\
\hline LA $5 \% 10 \mathrm{~min}$ & 19.5 & fg & 5.5 & $\mathrm{bc}$ & 12.5 & $\mathrm{C}$ \\
\hline LA $5 \% 30 \mathrm{~min}$ & 10.5 & c-e & 3.5 & $\mathrm{~b}$ & 7.0 & B \\
\hline LA $5 \% 60 \mathrm{~min}$ & 7.5 & $b-d$ & 0.5 & $\mathrm{a}$ & 4.0 & A \\
\hline Average & 58.2 & B & 23.0 & A & & \\
\hline \multicolumn{7}{|c|}{ Sample II } \\
\hline Control & 99.0 & $\mathrm{p}-\mathrm{r}$ & 91.0 & $\mathrm{n}$ & 95.0 & $\mathrm{~L}$ \\
\hline Fungicide & 36.5 & $\mathrm{gh}$ & 8.0 & $a-c$ & 22.3 & B \\
\hline W $10 \mathrm{~min}$ & 100.0 & $\mathrm{r}$ & 72.0 & $\mathrm{k}-\mathrm{m}$ & 86.0 & JK \\
\hline W $30 \mathrm{~min}$ & 99.5 & $\mathrm{qr}$ & 81.5 & $\mathrm{~m}$ & 85.5 & $\mathrm{KL}$ \\
\hline W 60 min & 98.0 & o-r & 77.0 & $1 \mathrm{~m}$ & 87.5 & I-K \\
\hline $\mathrm{H}_{2} \mathrm{O}_{2} 3 \% 10$ min & 92.0 & $\mathrm{n}$ & 48.5 & hi & 70.3 & $\mathrm{~F}$ \\
\hline $\mathrm{H}_{2} \mathrm{O}_{2} 3 \% 30 \mathrm{~min}$ & 68.5 & $\mathrm{j}-1$ & 35.5 & fg & 52.0 & $\mathrm{E}$ \\
\hline $\mathrm{H}_{2} \mathrm{O}_{2} 3 \% 60$ min & 57.0 & ij & 21.5 & $\mathrm{e}$ & 39.3 & D \\
\hline AA $1 \% 10 \mathrm{~min}$ & 100.0 & $\mathrm{r}$ & 76.5 & $1 \mathrm{~m}$ & 88.3 & KL \\
\hline AA $1 \% 30 \mathrm{~min}$ & 99.5 & $\mathrm{qr}$ & 62.5 & $\mathrm{jk}$ & 81.0 & H-J \\
\hline AA $1 \% 60 \mathrm{~min}$ & 99.0 & $\mathrm{p}-\mathrm{r}$ & 57.5 & $\mathrm{ij}$ & 78.3 & G-I \\
\hline AA $5 \% 10 \mathrm{~min}$ & 97.0 & $o-q$ & 64.5 & $\mathrm{jk}$ & 80.8 & G-I \\
\hline AA $5 \% 30 \mathrm{~min}$ & 96.0 & $n-p$ & 60.5 & $\mathrm{i}-\mathrm{k}$ & 78.3 & $\mathrm{GH}$ \\
\hline AA $5 \% 60 \mathrm{~min}$ & 94.5 & no & 59.5 & $\mathrm{i}-\mathrm{k}$ & 77.0 & FG \\
\hline LA $1 \% 10 \mathrm{~min}$ & 57.5 & $\mathrm{ij}$ & 20.5 & $\mathrm{e}$ & 39.0 & $\mathrm{D}$ \\
\hline LA $1 \% 30 \mathrm{~min}$ & 43.0 & gh & 16.0 & de & 29.5 & $\mathrm{C}$ \\
\hline LA $1 \% 60 \mathrm{~min}$ & 25.0 & ef & 15.0 & de & 20.0 & B \\
\hline LA 5\% $10 \mathrm{~min}$ & 11.0 & $\mathrm{~cd}$ & 4.0 & $a b$ & 7.5 & A \\
\hline LA $5 \% 30 \mathrm{~min}$ & 9.0 & $b-d$ & 3.0 & $\mathrm{a}$ & 6.0 & A \\
\hline LA $5 \% 60 \mathrm{~min}$ & 4.5 & $\mathrm{ab}$ & 3.0 & $a b$ & 3.8 & A \\
\hline Average & 69.3 & B & 43.9 & A & & \\
\hline
\end{tabular}

For explanation see Table 1

\section{Discussion}

Significant improvement of seed germination was observed in the experiment if the seeds were soaked in distilled water itself. This phenomenon suggested positive 
conditioning effect of the treatment but at the same time impeded assessment of the effects of hydrogen peroxide and organic acid seed treatment. On average after deterioration seeds of both samples germinated better than non-deteriorated. It was probably connected with decrease of seed infestation with fungi resulted in an increase of the number of normal seedlings and simultaneous decrease of the number of abnormal diseased seedlings. According to Maude [36] suboptimal storage conditions of seeds, such as high temperature and humidity, significantly affected survival of seedborne inoculum. However decline in viability of organisms with time generally does not cause a concomitant reduction in their infectivity.

In this experiment short-time treatment with 3\% hydrogen peroxide improved significantly germination of non-deteriorated seeds of sample II however, this beneficial effect was not observed in both samples after deterioration. The mode of action of hydrogen peroxide in the promotion of germination is unclear but may involve the scarification of the seedcoat [27] or oxidation of germination inhibitors [29]. Ogawa and Iwabuchi [29] suggested that the second mechanism is responsible for promoting germination of Z. elegans seeds. However, too high concentration of the hydrogen peroxide may negatively affected germination parameters. Sasaki et al. [24] found that the treatment of rice seeds with $\mathrm{H}_{2} \mathrm{O}_{2}$ at 5 to $100 \mathrm{mM}$ before sowing promoted seedling growth, whereas higher concentrations, 500 and $1000 \mathrm{mM}$ of $\mathrm{H}_{2} \mathrm{O}_{2}$ caused decrease of the shoot fresh weight. The authors observed also, that the treatment with $\mathrm{H}_{2} \mathrm{O}_{2}$ improved germination rate, causing rapid growth of seedlings in suboptimal conditions. Jaskani et al [26] reported that treating watermelon seeds with 1 and $2 \% \mathrm{H}_{2} \mathrm{O}_{2}$ enhanced seed germination and resulted in better seedling emergence. In present experiment hydrogen peroxide treatment prolonged germination time of deteriorated zinnia seeds, whereas had no influence on speed of germination of non-deteriorated seeds. Ascorbic acid several times, particularly after deterioration, positively affected germination. The phenomenon was probably connected with the antioxidative properties of this compound [9]. On the other hand lactic acid at concentration 5\%, and applied longer than $30 \mathrm{~min}$, negatively affected germination and vigour of zinnia seeds. However, beneficial effect of the treatment was maintained regardless of treatment time, if lower $1 \%$ concentration of this acid was used. Lang et al [37] also observed that soaking alfalfa seeds in 2.5 and $5 \%$ lactic acid solution for 30 min or longer significantly reduced percentage germination, whereas treating the seeds for $10 \mathrm{~min}$ had no negative effect on this parameter.

Lactic acid followed by hydrogen peroxide and ascorbic acid effectively limited growth of A. alternata, A. zinnia and Fusarium spp. on tested seeds. The antimicrobial properties of these compounds were reported by many authors [10, 12, 21, 30-32]. However, in terms of lactic acid, in general the effectiveness of different Lactobacillus strains has been studied, not just of the acid. Magnusson et al [14] observed antifungal activity of lactic acid bacteria against Aspergillus fumigatus, A. nidulans, Penicillium communae and Fusarium sporotrichioides, Rouse et al [17] proved effectiveness of the bacteria against Penicillium expansum, Laitila et al [13] against Fusarium aveanceum, F. culmorum, F. graminearum and $F$. oxysporum, and Sathe et al [16] against F. graminearum, Rhizopus stolonifer, Sclerotium oryzae, Rhizoctonia solani, Botrytis cinerea and Sclerotinia minor. De Muynck et al [15] suggested that organic acids are primarily responsible for the antifungal activity of lactic acid bacteria, and the results of present experiment entirely confirmed antimicrobial properties of lactic acid. 
Hydrogen peroxide treatment increased number of seeds free from fungi and decreased seed infestation with Alternaria spp. and Fusarium spp. however, the effect was more significant after deterioration. Peng and Kuc [38] observed that hydrogen peroxide at a very low concentration (2.61 $\left.10^{-5} \mathrm{M}\right)$ prevented germination of Peronospora tabacina sporangiospores, and Cladosporium cucumerinum and Colletotrichum lagenarium conidia in vitro. Moreover, peroxidase-generated hydrogen peroxide inhibited blue mould (P. tabacina) development on tobacco leaf disks. Authors suggested that $\mathrm{H}_{2} \mathrm{O}_{2}$ may function as an antifungal factor in disease resistance by direct inhibition of pathogens development, and/or by generation of other reactive free radicals that are antimicrobial.

Van der Wolf et al [11] observed that 2.5\% ascorbic acid reduced the frequency of fungal infected seeds of cabbage from 70 to ca. 30\%. At present experiment ascorbic acid, even at $5 \%$ concentration had no effect on the number of seeds free from fungi. Just the number of seeds infested with Fusarium spp. slightly decreased after the treatment however, the phenomenon can be connected with an increase of the seeds infestation with other fungi.

The negative, although interesting effect was an increase of seeds infestation with B. cinerea, observed in some cases after soaking zinnia seeds in hydrogen peroxide and organic acids solutions. These results are in contradiction to the reports that some antioxidants, including ascorbic acid, reduced development of grey mould disease [39, 40]. This phenomenon was probably caused by various factors. Low acidity, provided by organic acids (approximately $\mathrm{pH}$ 4.5-5.0, data not shown) favoured activity of endopolygalacturonase enzymes which are crucial in the infection process of this pathogen [41]. Additionally studies of Loewus [42] on oxalate production in vitro and in planta showed that L-ascorbic acid was the best carbon source for oxalic acid production in culture (in both mycelium and sclerotia). Whereas oxalic acid production by Sclerotinia sclerotiorum, a close relative of $B$. cinerea, plays a key role during initiation of infection by plant tissue, a synergistic process of cell wall maceration involving polygalacturonase. At last reduction of seeds infestation with other fungi could make space available for growth of $B$. cinerea. It can partially explain presence of this pathogen on treated seeds, even if the fungus was not detected in control.

The possibility of using natural compounds with GRAS (generally regarded as safe) status as a solution to seed treatment against fungal infection seems to be a promising option for organic production. However, the different sensitivity of the pathogen to the particular compounds indicates the need for further research including their effect on field emergence as well as their economic value.

\section{Conclusions}

1. Generally, treating zinnia seeds with organic acids more significantly affected seed germination and health after deterioration than before, and improvement of germination capacity was usually correlated with decrease of the percentage of abnormal diseased seedlings.

2. Deterioration had no influence on mean germination time, whereas in particular cases treating seeds with hydrogen peroxide and organic acids negatively affected this parameter.

3. On average higher number of seeds free from fungi, regardless of treatment, was observed after deterioration. 
4. Lactic acid followed by hydrogen peroxide and ascorbic acid effectively limited growth of A. alternata, A. zinnia and Fusarium spp. on/in zinnia seeds.

5. Generally, treating seeds with hydrogen peroxide and organic acids increased seeds infestation with $B$. cinerea.

\section{References}

[1] Kiecana I, Mielniczuk E. Acta Sci Pol, Hortorum Cultus. 2010;9(3):147-160.

http://wydawnictwo.up.lublin.pl/acta/hortorum_cultus/2010/acta_hort_9(3)_art_13.pdf.

[2] Gompert L, Windham M, Hamilton S. HortTechnology. 2001;11:71-74.

http://horttech.ashspublications.org/content/11/1/71.full.pdf+html.

[3] Szopińska D, Tylkowska S. Phytopathologia 2009;54:33-44.

http://www.au.poznan.pl/ptfit/pdf/P54/P54_04.pdf.

[4] Szopińska D, Tylkowska K, Deng ChJ, Gao Y. Seed Sci Technol. 2012;40:32-42.

http://www.ingentaconnect.com/content/ista/sst/2012/00000040/00000001/art00004.

[5] Wu WS, Yang YH. Plant Pathol Bull. 1992;1:115-123.

[6] Szopińska D. Folia Hort. 2014;26(1):19-29. DOI: 10.2478/fhort-2014-0002.

[7] Groot SPC, van der Wolf JM, Jalink H, Langerak CJ, van den Bulk RW. Seed Testing International. 2004;127:12-15. https://www.seedtest.org/upload/cms/user/STI127April2004.pdf.

[8] Gallagher RS, Fuerst EP. The ecophysiological basis of weed seed longevity in the soil. In: Basra AS, editor. Handbook of Seed Science and Technology. New York, London, Oxford: Food Products Press an Imprint of The Haworth Press, Inc.; 2006:521-557.

[9] Arrigoni O, De Tullio MC. Biochim Biophys Acta. 2002;1569:1-9. DOI: 10.1016/S0304-4165(01)00235-5.

[10] Molan PC. Bee World. 1992;73(1):5-28. http://hdl.handle.net/10289/2094.

[11] Van der Wolf JM, Birnbaum Y, van der Zouwen PS, Groot SPC. Seed Sci Technol. 2008;36:76-88. http://www.ingentaconnect.com/content/ista/sst/2008/00000036/00000001/art00008.

[12] Ricke SC. Poult Sci. 2003;82(4):632-639. DOI: 10.1093/ps/82.4.632.

[13] Laitila A, Alakomi H-L, Raaska L, Mattila-Sandholm T, Haikara A. J Appl Microbiol. 2002;93(4):566-576. DOI: 10.1046/j.1365-2672.2002.01731.x.

[14] Magnusson J, Ström K, Roos S, Sjögren J, Schnürer J. FEMS Microbiol Lett. 2003;219:129-135. DOI: 10.1016/S0378-1097(02)01207-7.

[15] Muynck C, Leroy AIJ, De Maeseneire S, Arnaut F, Soetaert W, Vandamme EJ. Microbiol Res. 2004;159(4):339-346. DOI: 10.1016/j.micres.2004.07.002.

[16] Sathe SJ, Nawani NN, Dhakephalkar PK, Kapadnis BP. J Appl Microbiol. 2007;103:2622-2628. DOI: 10.1111/j.1365-2672.2007.03525.x.

[17] Rouse S, Harnett D, Vaughan A, van Sinderen D. J Appl Microbiol. 2008;104:915-923. DOI: 10.1111/j.1365-2672.2007.03619.x.

[18] Voulgari K, Hatzikamari M, Delepoglou A, Georgakopoulos P, Litopoulou-Tzanetaki E, Tzanetakis N. Food Control 2010;21(2):136-142. DOI: 10.1016/j.foodcont.2009.04.007.

[19] Ström K, Schnürer J, Melin P. FEMS Microbiol Lett. 2005;246:119-124. DOI: 10.1016/j.femsle.2005.03.047.

[20] Lin MY, Yen ChL. J Agric Food Chem. 1999;47:1460-1466.

[21] Smilanick JL, Goates BJ, Denis-Arrue R, Simmons GF, Peterson GL, Henson DJ, et al. Plant Dis. 1994;78:861-865. DOI: 10.1094/PD-78-0861.

[22] Klein JD, Wood LA, Geneve RL. Acta Hort (ISHS). 2008;782:93-98. http://www.actahort.org/books/782/782_8.htm.

[23] Naredo MEB, Juliano AB, Lu BR, de Guzman F, Jackson MT. Seed Sci Technol. 1998;26:675-689.

[24] Sasaki K, Kishitani S, Abe F, Sato T. Plant Prod Sci. 2005;8(5):509-514. DOI: 10.1626/pps.8.509.

[25] Duval JR, NeSmith DS. HortScience. 2000;35(1):85-86. http://hortsci.ashspublications.org/content/35/1/85.short.

[26] Jaskani MJ, Kwon SW, Kim DH, Abbas H. Pak J Bot. 2006;38(1):89-98. http://www.pakbs.org/pjbot/PDFs/38(1)/PJB38(1)089.pdf.

[27] Chien CT, Lin TP. Seed Sci Technol. 1994;22:231-236.

[28] Conner PJ. HortScience. 2008;43(3):853-856. http://hortsci.ashspublications.org/content/43/3/853.full.

[29] Ogawa K, Iwabuchi M. Plant Cell Physiol. 2001;42(3):286-291. DOI: 10.1093/pcp/pce032. 
[30] Aharoni Y, Copel A, Fallik E. Ann Appl Biol. 1994;125:189-193. DOI: 10.1111/j.1744-7348.1994.tb04960.x.

[31] Fallik E, Aharoni Y, Grinberg S, Copel A, Klein JD. Crop Prot. 1994;13(6):451-454. DOI: 10.1016/0261-2194(94)90094-9.

[32] Sapers GM, Sites JE. J Food Sci. 2003;68(5):1793-1797. DOI: 10.1111/j.1365-2621.2003.tb12331.x.

[33] International Rules for Seed Testing. Switzerland, Bassersdorf: International Seed Testing Association; 2006.

[34] Jalink H, van der Schoor R. SeedCalculator 2.1. License number: 100200122. The Netherlands, Wageningen: Plant Res Int.; 1999.

[35] Mathur SB, Kongsdal O. Common laboratory seed health testing methods for detecting fungi. Switzerland, Bassersdorf: International Seed Testing Association; 2003.

[36] Maude RB. Seedborne diseases and their control. Principles \& Practice. UK, Wallingford: CAB International; 1996.

[37] Lang MM, Ingham $\mathrm{BH}$, Ingham SC. Int $\mathrm{J}$ Food Microbiol. 2000;58:73-82. DOI: 10.1016/S0168-1605(00)00297-X.

[38] Peng M, Kuc J. Phytopathology. 1992;82:696-699. http://www.apsnet.org/publications/phytopathology/ backissues/Documents/1992Articles/Phyto82n06_696.PDF.

[39] Elad Y. Plant Pathol. 1992;41(4):417-426. DOI: 10.1111/j.1365-3059.1992.tb02436.x.

[40] Tiedemann A. Physiol Mol Plant Pathol. 1997;50(3):151-166. DOI: 10.1006/pmpp/1996.0076.

[41] Kars I, Krooshof GH, Wagemakers L, Joosten R, Benen JAE, van Kan JAL. The Plant Journal 2005;43:213-225. DOI: 10.1111/j.1365-313X.2005.02436.x.

[42] Loewus FA. Phytochem. 1999;52:193-210. DOI: 10.1016/S0031-9422(99)00145-4.

\title{
OGRANICZENIE NEGATYWNYCH EFEKTÓW STARZENIA NASION Zinnia elegans Jacq. POPRZEZ TRAKTOWANIE NADTLENKIEM WODORU I KWASAMI ORGANICZNYMI
}

\author{
Katedra Fitopatologii i Nasiennictwa, Uniwersytet Przyrodniczy w Poznaniu
}

\begin{abstract}
Abstrakt: Pogorszenie jakości nasion podczas przechowywania jest ściśle związane z powstawaniem wolnych rodników. Niektóre związki o naturalnym pochodzeniu, znane ze swych właściwości antyoksydacyjnych i antygrzybowych, można potencjalnie wykorzystać do traktowania nasion w produkcji organicznej jako alternatywę dla pestycydów. Celem doświadczenia było określenie zdolności nadtlenku wodoru i kwasów organicznych do poprawy kiełkowania, wigoru i zdrowotności nasion cynii przed starzeniem i po starzeniu. Badano dwie próbki nasion cynii, odm. Illumination i Orys, różniące się wyjściowym zasiedleniem przez grzyby. W celu starzenia przetrzymywano nasiona przez $30 \mathrm{dni}$ w temperaturze $30^{\circ} \mathrm{C} \mathrm{i} \mathrm{w}$ warunkach $80 \%$ wilgotności względnej powietrza. Testy określające jakość nasion przeprowadzono przed i po starzeniu dla kontroli oraz nasion moczonych przez odpowiednio 10,30 i 60 min w 3,0\% roztworze nadtlenku wodoru oraz 1,0 i 5,0\% roztworach kwasów askorbinowego i mlekowego. Kontrole stanowiły nasiona nietraktowane, nasiona moczone w $0,2 \%$ roztworze preparatu Kaptan zawiesinowy $50 \mathrm{WP}$ przez 30 min oraz nasiona moczone w wodzie destylowanej przez odpowiednio 10, 30 i 60 min. Traktowanie nasion kwasami organicznymi w większym stopniu wpływało na nasiona po starzeniu niż przed, a poprawa zdolności kiełkowania związana była na ogół ze zmniejszeniem liczby kiełków anormalnych chorych. Starzenie nie miało wpływu na średni czas kiełkowania nasion, jakkolwiek w poszczególnych przypadkach traktowanie nasion nadtlenkiem wodoru i kwasami organicznymi niekorzystnie wpływało na ten parametr. Po starzeniu, bez względu na traktowanie, wzrastała liczba nasion wolnych od grzybów. Kwas mlekowy, a następnie nadtlenek wodoru i kwas askorbinowy efektywnie ograniczały wzrost A. alternata, A. zinnia i Fusarium spp. na nasionach cynii, jednak 5\% kwas mlekowy zastosowany dłużej niż $30 \mathrm{~min}$ negatywnie wpływał na kiełkowanie i wigor nasion. Traktowanie nasion nadtlenkiem wodoru i kwasami organicznymi niejednokrotnie zwiększało zasiedlenie nasion przez B. cinerea.
\end{abstract}

Słowa kluczowe: kwas askorbinowy, kwas mlekowy, nadtlenek wodoru, starzenie nasion, cynia 